\title{
Determining the effective number and surfaces of teeth for forensic dental identification through the 3D point cloud data analysis
}

Arofi Kurniawan ${ }^{1,2^{*}}$ D, Kouya Yodokawa ${ }^{3}$, Moe Kosaka ${ }^{1}$, Koichi Ito ${ }^{3}$, Keiichi Sasaki ${ }^{1,4}$, Takafumi Aoki ${ }^{3}$ and Toshihiko Suzuki ${ }^{1}$

\begin{abstract}
Background: The assimilation between three-dimensional (3D) imaging techniques and dental forensic science can provide rich and stable information for human identification. This study aimed to determine the effective number and surfaces of teeth for dental identification through the 3D imaging approach.

Material and methods: In the present study, maxillary dental casts were fabricated from subjects who met the inclusion criteria and scanned using a 3D scanner Vivid 910. Rapidform XOS/SCAN software was used to create and trim the 3D point cloud data. Subsequently, two types of 3D surface data of dental casts were registered and the root mean square errors (RMSEs) between subjects were calculated using iterative closest point (ICP) algorithm in MATLAB. Two sets of experiments with 120 combinations of the superimposed 3D dataset were designed, termed as experiments 1 and 2 .
\end{abstract}

Results: In experiment 1, the difference between subjects was clearly distinguished with a minimum of six teeth of the dental arch. The results of experiment 2 suggest that the labial surfaces of the anterior teeth are sufficient to be used for dental identification.

Conclusion: Through these experiments for all possible pairs of subjects, a clear difference was observed in the RMSE between the genuine and imposter pairs. These results indicate the potential of using the 3D imaging technique to achieve highly accurate human identification. It is suggested that a future study with a larger sample number will evaluate the robustness and accuracy of this method.

Keywords: Forensic science, Forensic odontology, Personal identification, Root mean square error, Threedimensional imaging

\section{Background}

Teeth are the hardest and most durable substance in the human body that can endure extreme conditions, are resistant to decomposition, and have various anatomical characteristics. In forensics fields, the unique characteristics of human dentition are widely accepted for identification. Because of these advantages, forensic odontology is

\footnotetext{
* Correspondence: arofi.kurniawan@fkg.unair.ac.id

1 Division of Dental and Digital Forensics, Graduate School of Dentistry,

Tohoku University, Sendai 980-8575, Japan

2Department of Forensic Odontology, Faculty of Dental Medicine, Universitas Airlangga, Surabaya 60132, Indonesia

Full list of author information is available at the end of the article
}

considered as a dependable and reliable method of human identification (Andersen et al. 1995; Delattre 2000; Pretty and Sweet 2001; Koehler and Brown 2009; Verma et al. 2014; Priyanka and Prasad 2015).

Several scientific analysis and data collection methods have undergone a significant transformation in forensic odontology to accommodate human identification (Jeddy et al. 2017). One of the great improvements in this area is the assimilation between forensic dentistry and information technology such as dental imaging techniques, bite-mark analysis, and preservation of the dental records (Heinrich et al. 2018). The common digital 
imaging technique which is used in dental identification is carried out using the two-dimensional (2D) approach, such as comparisons of radiographic images, photographs, and visual identification (Manigandan et al. 2015). In addition to $2 \mathrm{D}$ imaging, many studies use the three-dimensional (3D) imaging systems to obtain more accurate and precise results in forensic identification (Evans et al. 2010; Pramod et al. 2012; Abduo and Bennamoun 2013; Ata-Ali and Ata-Ali 2014).

Many 3D image devices and software have been developed to achieve more robust forensic evidence for courts and medico-legal purposes. The $3 \mathrm{D}$ imaging approach in forensic identification offers documentation, analysis, and visualization of dental evidence with minimum dimensional distortion (Thali et al. 2003; Marques et al. 2013; Chaudhary et al. 2018). A previous study by Evans et al. (2013) found that the 3D scanner Vivid 910 (Konica Minolta Sensing, Inc., Tokyo, Japan), also used in this study, was adequately recorded the surface of the dental casts.

This study was conducted by examining the 3D surface data of dental casts obtained from 3D scanner Vivid 910 in two experimental designs. The first experiment was aimed to verify the effective number of teeth and the involved dental arch segment, while the second experiment was arranged to determine the minimum surface of tooth representing and being used sufficiently for dental identification.

\section{Materials and methods}

\section{Dental cast acquisitions}

Subjects who met the inclusion criteria have engaged in this study after voluntarily providing written informed consent. The inclusion criteria of this study were healthy subjects without severe dental crowding, dental caries, attrition/abrasion, missing teeth, and orthodontic appliances, and agreed to participate as a subject. All experiment designs were conducted in accordance with a protocol reviewed by the Independent Ethics Committee of Tohoku University School of Dentistry, Japan (permit no: 2017-317). The number of subjects enrolled in this study was determined after consulting an expert in statistics.

The maxillary dental arch was considered to be used in this study because it is easier for forensic dentists to reach and record in the actual identification cases. For example, the complication sometimes arises when opening the mouth of the dead body. In such situations, the labial surface of maxillary teeth will be easier to reach and register than the mandibular teeth.

The maxillary dental condition of the participant was recorded using a dental impression tray and the alginate impression material powder Aroma Fine Mixer Type (GC Corp., Tokyo, Japan) to fabricate the dental cast. Afterward, each dental cast was trimmed and cleaned on its surfaces from small bubbles to obtain optimum scanning results.

\section{D scanner and software}

The 3D surface data collection of the dental cast in this study was performed using a 3D scanner Vivid 910. Vivid 910 is a non-contact 3D digitizer that employs a triangulation method. The Vivid scanner has been used for several years by clinical and forensic applications, such as examining changes in patients with cleft palates (Kitagawa et al. 2004), investigating facial changes (Kau and Richmond 2008), and analyzing bite-mark evidence (Flora et al. 2009; Evans et al. 2013).

The Polygon Editing Tool (PET) software that comes with Vivid 910 was used to align and merge multiple scanned data into a single polygonal model. The polygonal model acquired from the PET then processed in the Rapidform XOS/SCAN software (INUS Technology, Inc., Seoul, South Korea) to generate the 3D point cloud data (Fig. 1).

\section{D image registration}

The dental cast scanning process was done by placing the dental cast on a rotating table to simplify its 360degree rotation, with the biting edges facing upwards. Vivid 910 positions towards the dental cast was approximately $45^{\circ}$ allowing the scanning of the entire dental cast surfaces. Each dental cast was scanned from six points of view (a field of approximately $60^{\circ}$ could be captured in a single scan), resulting in a single $3 \mathrm{D}$ data from each point of view. The dental cast scanning was done twice in separate sessions.

Some algorithms have been developed in several studies to automatically align and estimate the similarities between two 3D images in a process commonly called image registration (Williams and Bennamoun 2001; Williams et al. 2003; Mian et al. 2006). A similar approach of the image registration was used in this study, starting with the adjustment of the transformation parameters of each scanned 3D data in PET software, i.e., $3 \mathrm{D}$ rotation and translation.

After the adjustment process in PET, all of the scanned 3D data were processed using the Rapidform XOS/SCAN software. Multiple 3D data acquired from six points of view were aligned for each subject based on the transformation parameters obtained by PET and merged into a single $3 \mathrm{D}$ dataset, resulting in $3 \mathrm{D}$ point cloud data.

Two sets of experiments were designed by superimposing the genuine and the imposter pairs, termed as experiment 1 and experiment 2 (Fig. 2). The genuine pair is a concept of information science to define the superimposition between two 3D datasets derived from the same subjects from the first and the second scanning. 


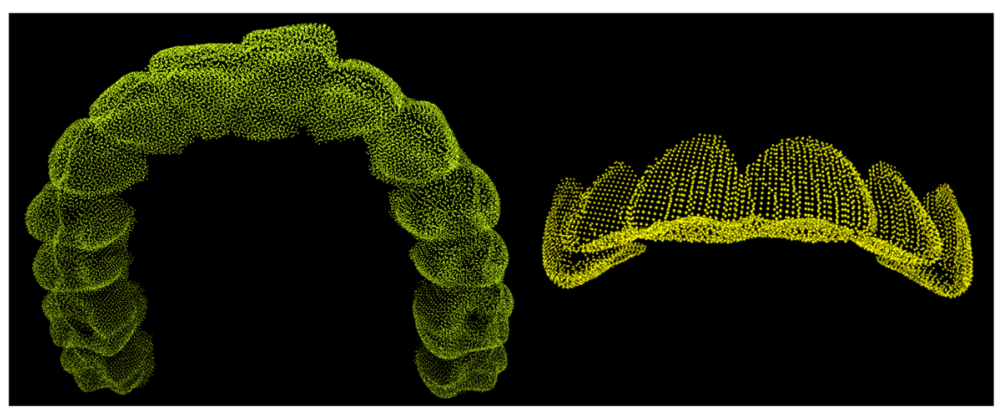

Fig. 1 Preview of the point clouds of the 3D dataset of dental casts

The imposter pair is the superimposition of two 3D datasets from the different subjects, i.e., subject 1 (first scanning) vs subject 2 (second scanning).

The 3D dataset superimposing groups were classified as follows: eight groups in experiment 1 and three groups in experiment 2. Afterward, 3D datasets between subjects (both the genuine and the imposter pairs) were aligned following the midline of the reference image as the guideline. Especially, for the right and left posterior groups in experiment 1 , the $3 \mathrm{D}$ dataset alignment was based on the position of the second premolar teeth of the reference images. The $3 \mathrm{D}$ dataset from the first scan was used as the reference images, and the 3D dataset from the second scan was referred to as the moving images.

Root mean square errors (RMSEs) in both experiments were calculated using the iterative closest point (ICP) algorithm, performed in MATLAB (MathWorks, Inc., Natick, MA, USA). The ICP is an algorithm employed to minimize the discrepancies between two $3 \mathrm{D}$ data point clouds (Fig. 3). The RMSE can be defined as the representation of the standard deviation of the discrepancies between the observed subjects (Zhang 2014). The RMSE distribution patterns of each group were investigated in experiments 1 and 2 . An overview of $3 \mathrm{D}$ data

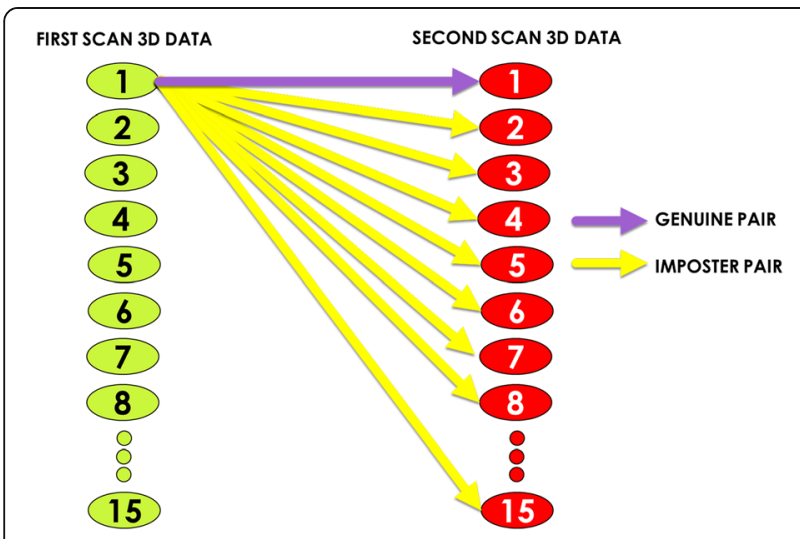

Fig. 2 A brief description of the genuine and the imposter pairs acquisition, processing, and data analysis are explained in Fig. 4.

\section{Experimental design \\ Experiment 1}

The first experiment was performed to verify the effective number of teeth and the involved dental arch segment for forensic identification. All surfaces of teeth (labial, palatal, and occlusal) were included in experiment 1.

The groups made for 3D dataset superimposition in experiment 1 were classified as follows: full arch (A); partial arch from the left to the right second incisor (B), the left to the right canine $(C)$, the left to the right first premolar (D), the right central incisor to the right last molar (E), the left central incisor to the left last molar (F), the right second premolar to the right last molar (G), and the left second premolar to the left last molar $(\mathrm{H})$. The number of included teeth and the involved dental arch segment (bilateral and unilateral arches) for each group are illustrated in Fig. 5.

\section{Experiment 2}

The second experiment was conducted by superimposing the labial surfaces of the anterior teeth between subjects. The labial surface was selected as an approach to the development of actual identification tasks, i.e., when there are difficulties in opening the victim's mouth, recording the labial surfaces of the anterior teeth will be easier for the forensic dentist. The 3D point cloud data of the anterior teeth and bilateral dental arch groups (groups B, C, and D in experiment 1) were divided by

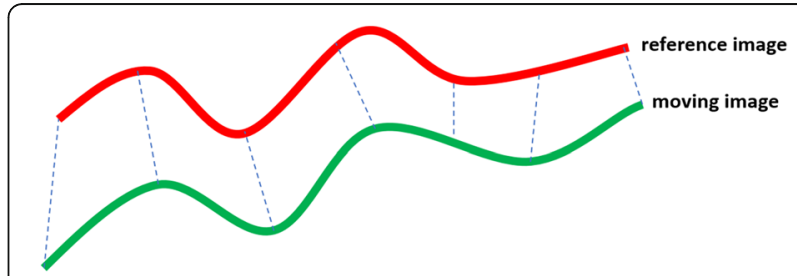

Fig. 3 The basic concept explanation of the iterative closest point (ICP) algorithm 


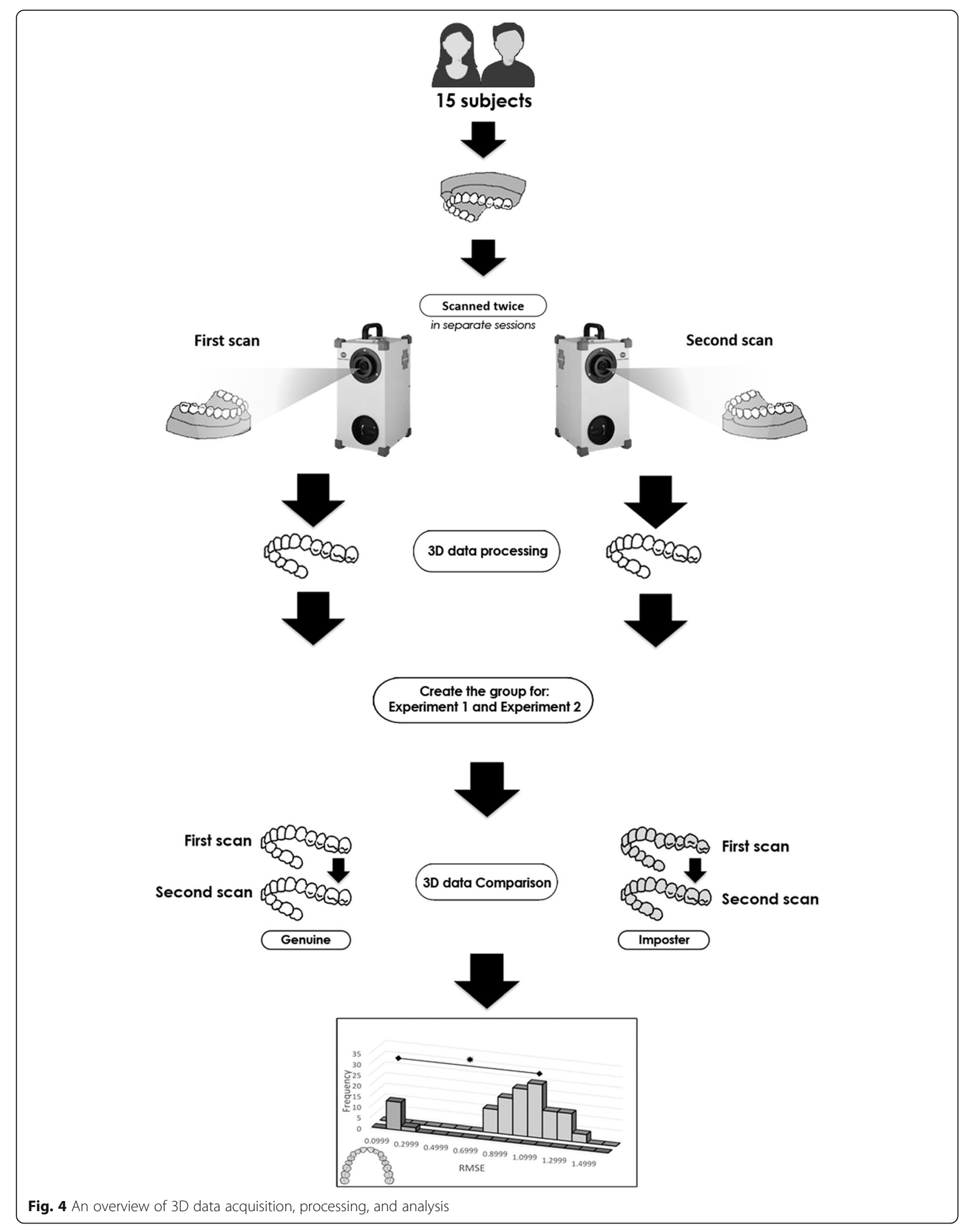




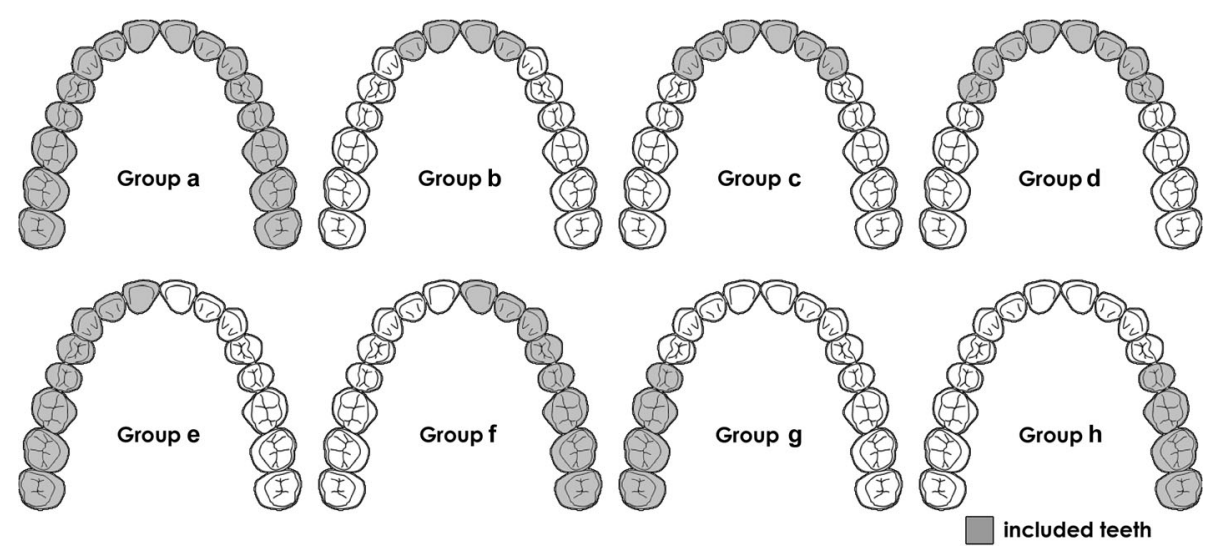

Fig. 5 Group s for superimposing the 3D dataset of the dental casts in experiment 1. Full arch (a); partial arch from the left to the right second incisor (b), the left to the right canine (c), the left to the right first premolar (d), the right central incisor to the right last molar (e), the left central incisor to the left last molar (f), the right second premolar to the right last molar $(\mathbf{g})$, and the left second premolar to the left last molar (h)

excluding the palatal surfaces and retaining only the labial surfaces. Groups for experiment 2 (Fig. 6) were classified as follows: labial surfaces from the right to the left second incisor $\left(\mathrm{B}^{\prime}\right)$, the right to the left canine $\left(\mathrm{C}^{\prime}\right)$, and the right to the left first premolar $\left(D^{\prime}\right)$.

\section{Statistical analysis}

The statistical analysis in this study was carried out using IBM SPSS Statistics version 23.0 (International Business Machines Corp., Armonk, NY, USA). The scattered plot diagram described the distribution of the RMSE value of the genuine and the imposter pairs. The Mann-Whitney U test was used to analyze the significance of the difference of the RMSE value between the genuine and imposter pairs, and between experiments 1 and 2 . The threshold of statistical significance was set at $p<0.05$.

\section{Results}

\section{Experiment 1}

Table 1 shows a summary of the descriptive analysis of experiment 1. In general, the mean and SD of the RMSE

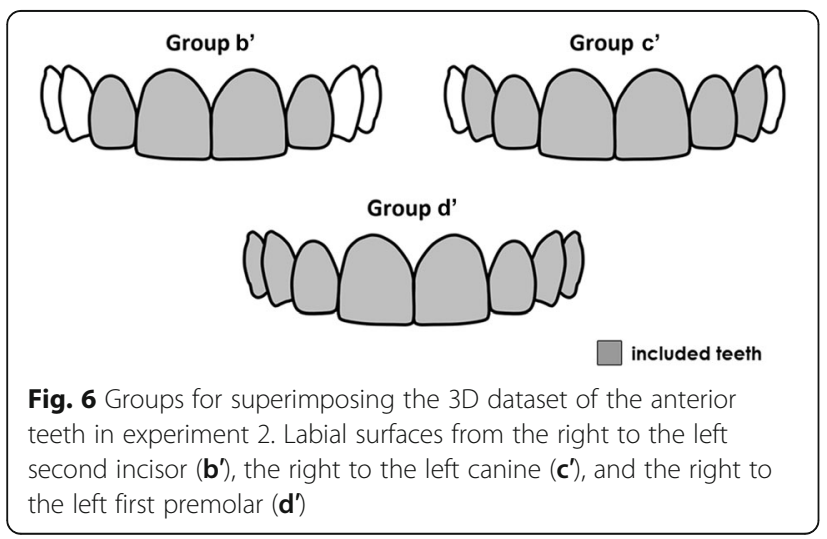

value of the imposter pairs were significantly larger than the observed values in the genuine pairs. The quantitative analysis was revealed that the average RMSE value of the genuine pair was $0.182 \pm 0.032 \mathrm{~mm}$, while that of the imposter pair was $0.766 \pm 0.149 \mathrm{~mm}$. The findings of the Mann-Whitney $U$ test indicated a significant difference between the genuine and imposter pairs in the mean and SD of the RMSE, with $p<0.05$.

The difference between the genuine and the imposter pairs was described in the scattered plot diagram and the histogram as shown in Figs. 7 and 8. The distribution pattern of the genuine and imposter pairs in group A, comprising of the full dental arch (16 teeth), was

Table 1 Descriptive statistics of the genuine and the imposter pairs in experiment 1

\begin{tabular}{llll}
\hline Group & Pair & RMSE mean $(\mathrm{mm})$ & SD \\
\hline A & Genuine & 0.184 & 0.029 \\
& Imposter & 1.016 & 0.157 \\
B & Genuine & 0.176 & 0.036 \\
& Imposter & 0.660 & 0.192 \\
C & Genuine & 0.176 & 0.033 \\
& Imposter & 0.782 & 0.199 \\
D & Genuine & 0.181 & 0.032 \\
& Imposter & 0.875 & 0.204 \\
E & Genuine & 0.188 & 0.033 \\
& Imposter & 0.828 & 0.154 \\
F & Genuine & 0.181 & 0.030 \\
& Imposter & 0.805 & 0.155 \\
G & Genuine & 0.189 & 0.033 \\
& Imposter & 0.574 & 0.142 \\
H & Genuine & 0.182 & 0.028 \\
& Imposter & 0.588 & 0.153 \\
\hline
\end{tabular}



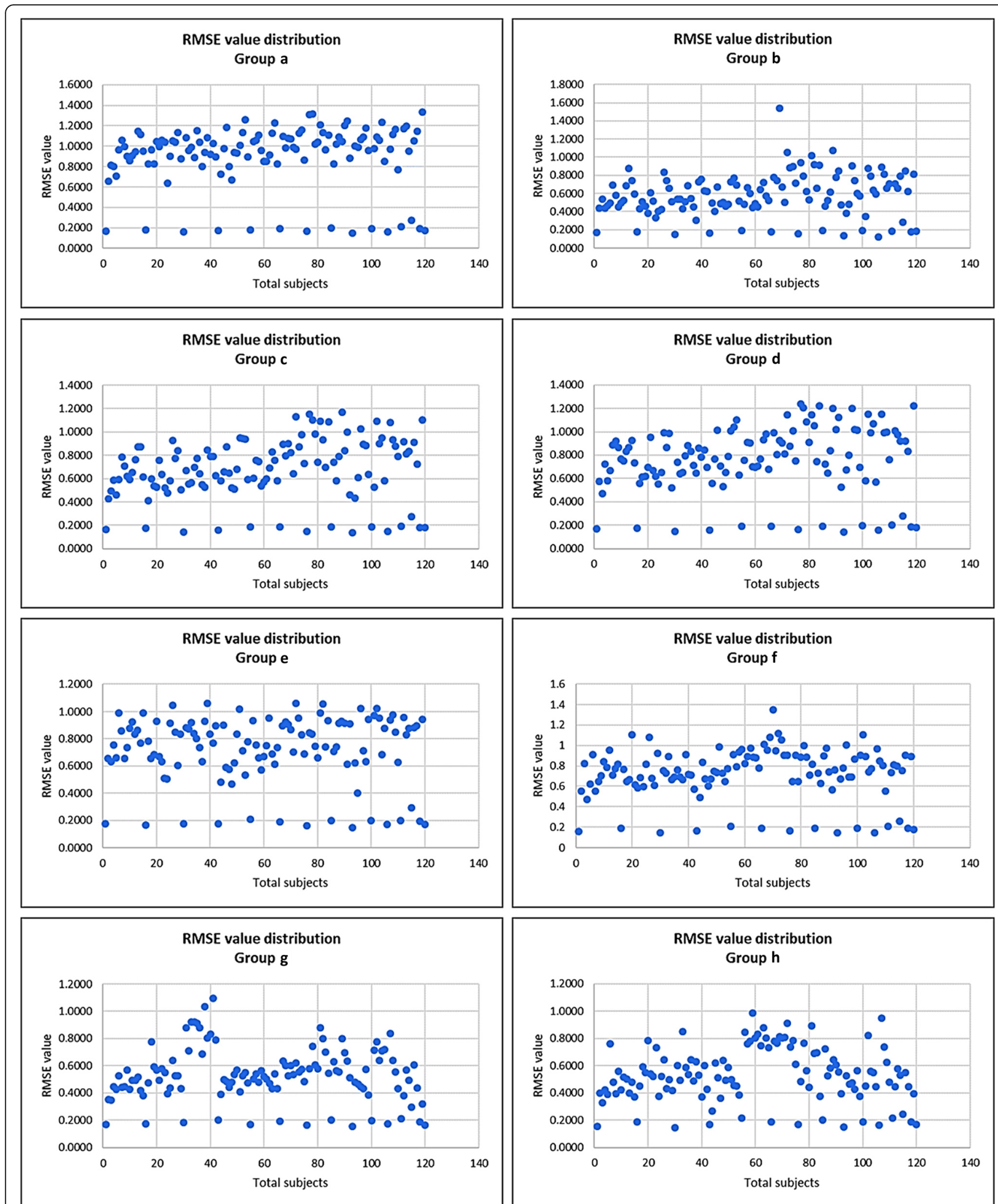

Fig. 7 Scattered plot diagram of experiment 1 (Groups $\mathbf{a}-\mathbf{h}$ ). Full arch (a); partial arch from the left to the right second incisor (b), the left to the right canine (c), the left to the right first premolar (d), the right central incisor to the right last molar (e), the left central incisor to the left last molar (f), the right second premolar to the right last molar ( $\mathbf{g}$ ), and the left second premolar to the left last molar (h) 

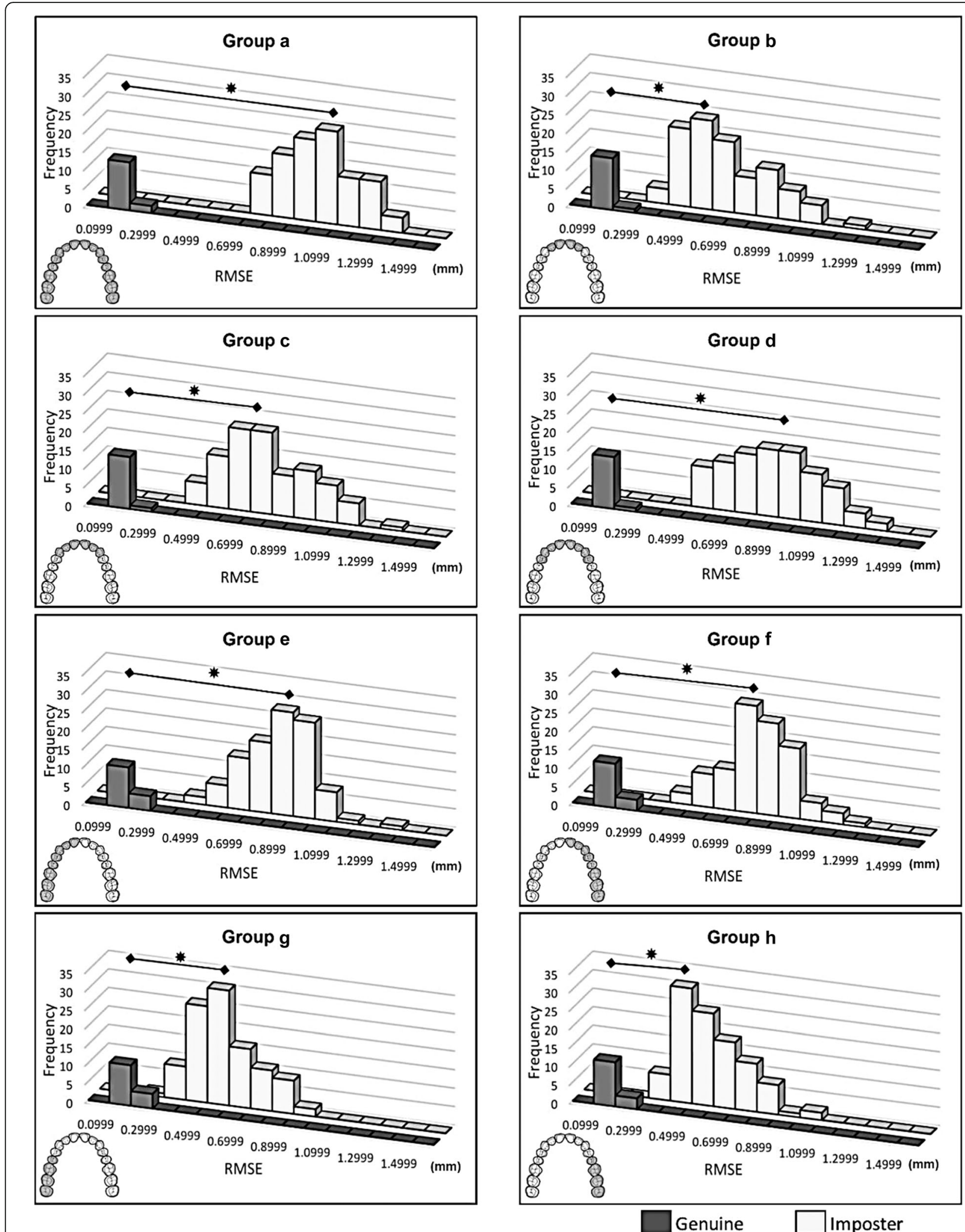

Fig. 8 The distribution pattern of root mean square errors (RMSEs) of the three-dimensional data for the genuine and imposter pairs in experiment 1 (Groups $\mathbf{a}-\mathbf{h}$ ) 
apparent that the histogram limit was in the wide range for the imposter pair compared with the genuine pair, and the RMSE value of the genuine pairs was in the lower level of the scattered plot diagram. On the other hand, the peak of the histogram and the RMSE value distribution of groups $\mathrm{C}, \mathrm{D}, \mathrm{E}$, and $\mathrm{F}$ on the scattered plot diagram and histogram had narrower, between the genuine and the imposter pairs. There were also clear differences in these groups, with 6 teeth in group $C$ and 8 teeth in groups D, E, and F. In groups B, G, and H, consisting of 4 teeth, few overlapping areas on the histogram were observed. Even there were a few overlapping areas in the distribution; there were possibilities for distinguishing between the genuine and the imposter pair in these groups.

\section{Experiment 2}

Experiment 2 included only the labial aspect of teeth. The descriptive analysis as defined in Table 2 shows that the mean and SD of the RMSE value in the genuine pairs were smaller than in imposter pairs. The genuine pairs had an average RMSE value of $0.187 \pm 0.041 \mathrm{~mm}$, while the imposter pairs were $0.783 \pm 0.219 \mathrm{~mm}$. According to the Mann-Whitney $U$ test results, significant differences between the genuine and the imposter pairs were also found in all groups, with $p<0.05$.

Similar to the findings of experiment 1 , the differences in the distribution pattern of the genuine and imposter pairs were also observed in these three groups (Fig. 9). Group B' consisted of four teeth and also showed significant differences between the genuine and imposter pairs; however, the distribution had a few overlapping areas. The Mann-Whitney $U$ test revealed that there were no significant differences in the RMSE between the labial (groups $\mathrm{B}^{\prime}, \mathrm{C}^{\prime}$, and $\mathrm{D}^{\prime}$ ) and full teeth surfaces (groups $\mathrm{B}, \mathrm{C}$, and $\mathrm{D}$ ), $p>0.05$.

\section{Discussion}

Three-dimensional surface matching is a process of comparing the surface and decides the best match between two given similar surfaces. Considering many advantages of $3 \mathrm{D}$ imaging techniques, such as accuracy and precision, the $3 \mathrm{D}$ surface matching was used in this

Table 2 Descriptive statistics of the genuine and the imposter pairs in experiment 2

\begin{tabular}{llll}
\hline Group & Pair & RMSE mean $(\mathrm{mm})$ & SD \\
\hline B & Genuine & 0.189 & 0.045 \\
& Imposter & 0.652 & 0.193 \\
C & Genuine & 0.185 & 0.040 \\
& Imposter & 0.800 & 0.235 \\
D & Genuine & 0.186 & 0.038 \\
& Imposter & 0.896 & 0.230 \\
\hline
\end{tabular}

study by calculating the RMSE value (Besl and McKay 1992; Williams et al. 2003; Mian et al. 2006; Xiao et al. 2007).

Considerable interest has been shown in the development of 3D imaging for forensic identification. Evans et al. (2010) performed an experiment to explore the potential of 3D imaging in forensic odontology and simulated a 3D imaging bite-mark analysis using the MAVIS stereo-photogrammetric system and Vivid 910. According to the study by Evans et al., Vivid 910, which is also used in this study, has produced an excellent 3D image data of dental casts (Evans et al. 2010, 2013).

The objective of this study was to determine the effective number of teeth used in personal identification by analyzing the 3D surface data of dental casts. Therefore, subjects without severe dental crowding, attrition/ abrasion, or missing teeth were enrolled to establish a suitable condition of the dental cast for the classification groups. Eight groups (groups $\mathrm{A}-\mathrm{H}$ ) in experiment 1 were classified to verify the effective number of teeth and the dental arch section used for identification. Fullsurface 3D data of dental cast was involved in experiment 1 to examine the effectivity of this method. Then, the area for comparing the $3 \mathrm{D}$ dataset was limited to the labial aspect in experiment 2 to specify the effective tooth surface for dental identification work.

In experiment 1 , the RMSE value of the imposter pairs showed significantly wider ranges than the genuine pairs and the difference between the genuine and the imposter pair were described on the color map of the superimposed 3D dataset (Figs. 10 and 11). Significant differences in RMSE between the imposter and genuine pairs were observed in all eight groups, suggesting several useful features of the condition. First, the number of included teeth influences the distribution pattern of RMSE between the genuine and imposter pairs. The difference between the genuine and imposter pairs was clearly distinguished by comparing at least six teeth. While the entire-arch presentation enhances overall reliability, the identification process can be applied to a segment of the dental arch. Second, when more teeth were included in the comparison, the histogram peak had a wider range in the imposter pair. On the contrary, when fewer teeth were included, the distribution of the imposter pair had a narrower limit than the genuine pair. Third, the involved dental arch segment has also affected the distribution of RMSE value. The results from groups involving both the left and right dental arches (bilateral arches) were clearer when distinguishing between the genuine and imposter pairs compared to the use of onesided dental arches (unilateral arches). The authors believe that there are fewer differences when superimposing fewer teeth and considering a small section of the dental arch with subjects who meet the inclusion criteria. 


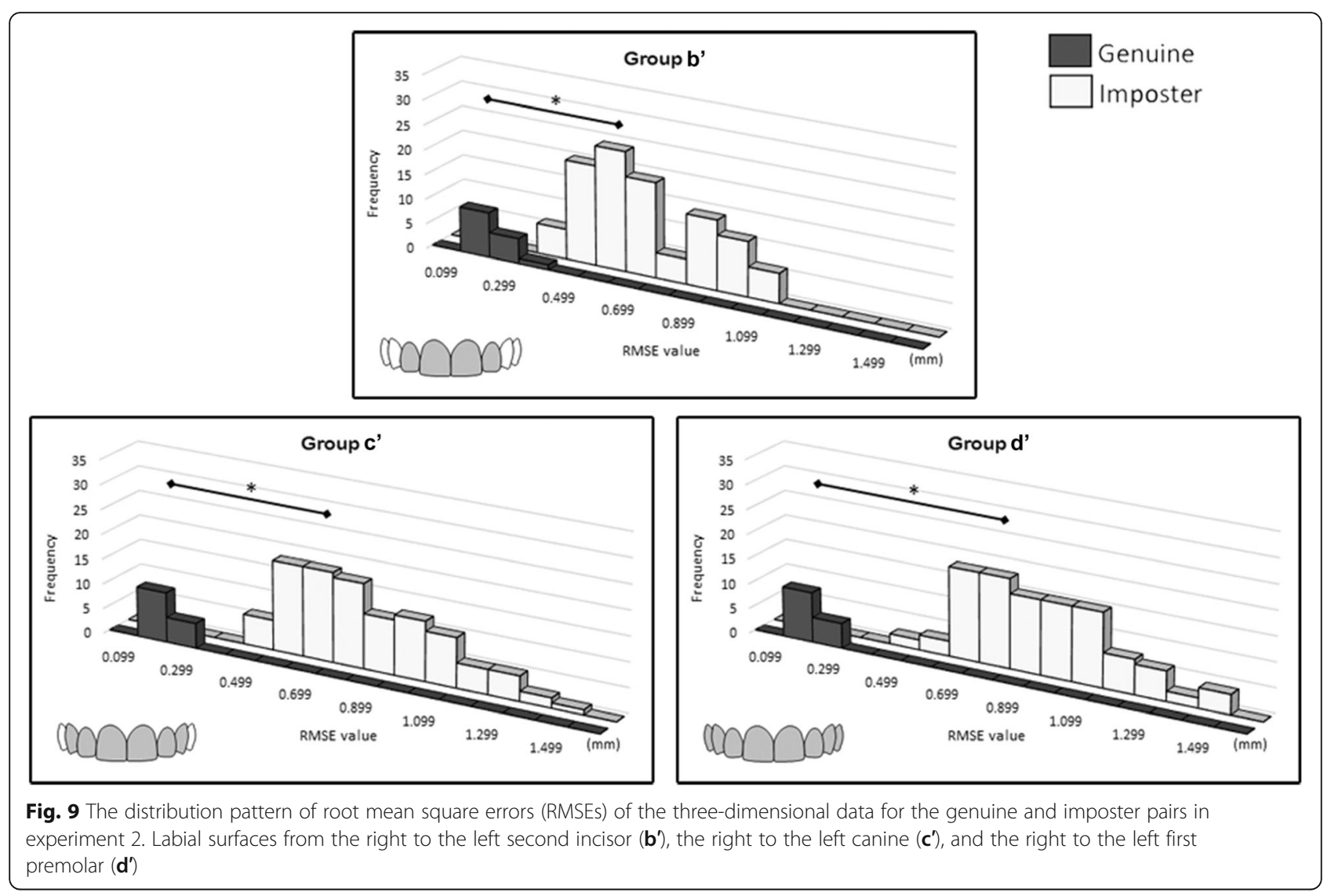

In the actual identification process, the forensic team is facing a major challenge in establishing the identity of the victim correctly. In some cases, the victim's intraoral state may not be well preserved, and therefore, forensic dentists are unable to determine the number of remaining teeth of the victim. There are also problems or obstacles in opening the victim's mouth in some mortality cases and may prevent the forensic dentist from recording the intraoral condition precisely. Even in such situations, it is possible to obtain a dental impression of the labial surface of the anterior teeth using some dental materials.

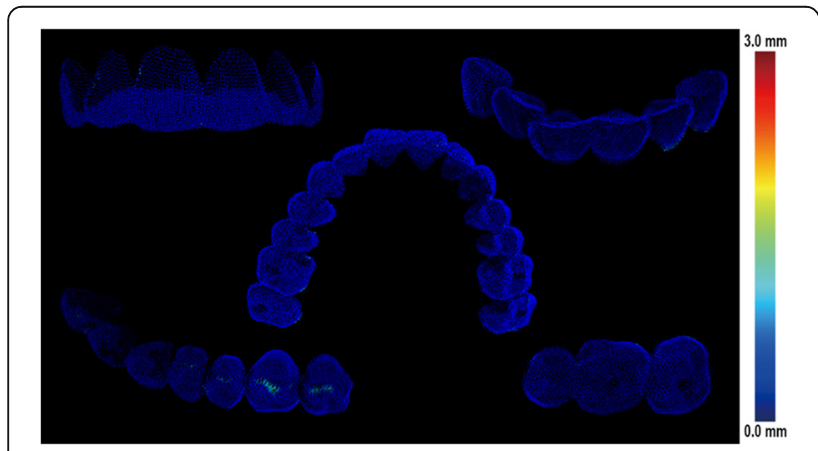

Fig. 10 Color map of the superimposed 3D dataset within the genuine pairs
Relying on the accuracy and precision of the 3D imaging system, useful and adequate information could be obtained for dental identification work even if the image is limited to the labial surface of the anterior teeth. In the present study, the result of experiment 1 was used to generate further limited conditions for experiment 2 to examine the effectiveness of the method to be applied in actual identification tasks, considering the cases with mouth-opening limitations.

Significant differences between the genuine and imposter pairs were also observed in the superimposition

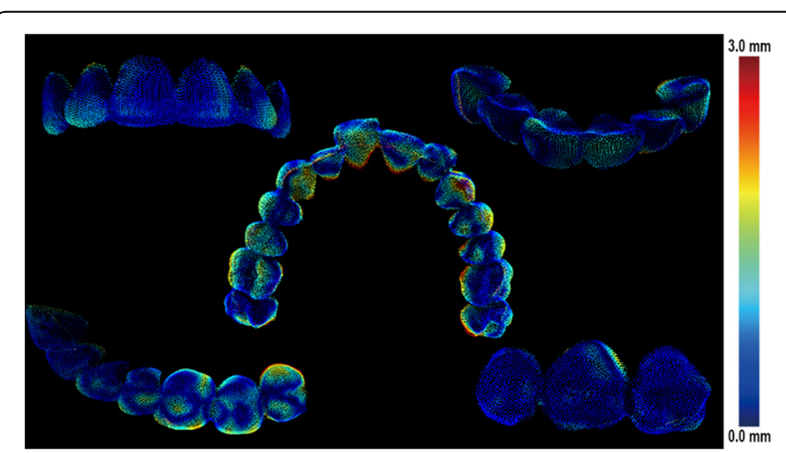

Fig. 11 Color map of the superimposed 3D dataset of the imposter pairs 
of the labial surface in all three groups. Nonetheless, similar to the result of experiment 1 , when more than 6 teeth were included, the differences were more noticeable. Besides, there were no significant differences in the RMSE values between experiments 1 and 2. These results suggest that the $3 \mathrm{D}$ images of a minimum six labial surface of the anterior teeth are adequate to be used for dental identification tasks.

In the actual identification work, it is much easier for the forensic dentist to register, taking an impression, and acquiring the 3D data of the anterior teeth. This method could, therefore, be applied not only to trismus cases, but also to cases involving missing molars, artificial crown coverage, no dental treatment (sound teeth), and minimizing a dental autopsy to the dead body. The curve shape of the dental arch pattern of the anterior teeth is also an advantage when analyzing the 3D data of the dental arch using the ICP algorithm since it is easier to be aligned between subjects.

To avoid inter-observer errors, one researcher semiautomatically performed the scanning, data processing, superimposing, and the pair value calculation. Therefore, optimal data acquisition at the scanning process has to be encouraged obtaining precise 3D data.

\section{Conclusion}

Through these experiments for all possible pairs of subjects, a clear difference in the RMSE was observed between the genuine and imposter pairs. These results indicate the ability for highly accurate human identification using the 3D imaging technique. The future study with a greater number of samples will be suggested to assess the robustness and the reliability of this method.

Although some helpful conditions for 3D dental identification have been suggested in this study, further studies are necessary to improve the reliability of this method for a specific identification task, which may involve cases of random missing teeth, extreme crowding, fractured teeth, anomaly, etc. Moreover, it is important to compare the present study approach with the use of an intra-oral scanner that has been developed rapidly in recent years and endeavors to devise a simple and easy method that can be applied in actual daily work.

\section{Abbreviations}

2D: Two-dimensional; 3D: Three-dimensional; ICP: Iterative closest point; PET: Polygon editing tool; RMSE: Root mean square error

\section{Acknowledgements}

The authors thank Dr. Jun Aida, Department of International and Community Oral Health, Graduate School of Dentistry, Tohoku University for his advices in the statistical analysis of this study.

\section{Authors' contributions}

$A K, K Y$, and MK contributed to the dental cast acquisition and scanning. AK and $K Y$ contributed to 3D image processing and RMSE calculation. KY, TA, and $\mathrm{Kl}$ contributed to arrange the programming language in Matlab. AK, KS,
$\mathrm{TS}, \mathrm{Kl}$, and TA contributed to the study conception and design. AK and MK contributed to the drafting of the manuscript. AK, MK, TS, and KI contributed to the critical revision of the manuscript. All authors have read and approved the final manuscript.

\section{Availability of data and materials}

Not applicable.

\section{Ethics approval and consent to participate}

Ethical approval has been obtained from the Independent Ethics Committee of Tohoku University School of Dentistry, Japan (permit no. 2017-3-17).

\section{Consent for publication}

Consent to publish this study is given by all the authors.

\section{Competing interests}

The authors declare that they have no competing interests.

\section{Author details}

'Division of Dental and Digital Forensics, Graduate School of Dentistry, Tohoku University, Sendai 980-8575, Japan. ²Department of Forensic Odontology, Faculty of Dental Medicine, Universitas Airlangga, Surabaya 60132, Indonesia. 'Department of Computer and Mathematical Sciences, Graduate School of Information Sciences, Tohoku University, Sendai 980-8579, Japan. ${ }^{4}$ Division of Advanced Prosthetic Dentistry, Graduate School of Dentistry, Tohoku University, Sendai 980-8575, Japan.

Received: 3 September 2019 Accepted: 28 January 2020

Published online: 06 February 2020

\section{References}

Abduo J, Bennamoun M (2013) Three-dimensional image registration as a tool for forensic odontology: a preliminary investigation. Am J Forensic Med Pathol 34:260-266. https://doi.org/10.1097/PAF.0b013e31829f6a29

Andersen L, Juhl M, Solheim T, Borrman H (1995) Odontological identification of fire victims--potentialities and limitations. Int J Legal Med 107:229-234

Ata-Ali J, Ata-Ali F (2014) Forensic dentistry in human identification: a review of the literature. J Clin Exp Dent 6:e162-e167. https://doi.org/10.4317/jced. 51387

Besl PJ, McKay ND (1992) A method for registration of 3-D shapes. IEEE Trans Pattern Anal Mach Intell. https://doi.org/10.1109/34.121791

Chaudhary R, Doggalli N, Chandrakant H, Patil K (2018) Current and evolving applications of three-dimensional printing in forensic odontology: a review. Int J Forensic Odontol 3:59-65. https://doi.org/10.4103/ijfo.ijfo_28_18

Delattre VF (2000) Burned beyond recognition: systematic approach to the denta identification of charred human remains. J Forensic Sci 45:589-596

Evans S, Jones C, Plassmann P (2010) 3D imaging in forensic odontology. J Vis Commun Med 33:63-68. https://doi.org/10.3109/17453054.2010.481780

Evans ST, Jones C, Plassmann P (2013) 3D imaging for bite mark analysis. Imaging Sci J 61:351-360. https://doi.org/10.1179/1743131X11Y.0000000054

Flora G, Tuceryan M, Blitzer H (2009) Forensic bite mark identification using image processing methods

Heinrich A, Guttler F, Wendt S et al (2018) Forensic odontology: automatic identification of persons comparing antemortem and postmortem panoramic radiographs using computer vision. Rofo 190:1152-1158. https:// doi.org/10.1055/a-0632-4744

Jeddy N, Ravi S, Radhika T (2017) Current trends in forensic odontology. J Forensic Dent Sci. https://doi.org/10.4103/jfo.jfds_85_16

Kau CH, Richmond S (2008) Three-dimensional analysis of facial morphology surface changes in untreated children from 12 to 14 years of age. Am J Orthod Dentofac Orthop. https://doi.org/10.1016/j.ajodo.2007.01.037

Kitagawa T, Kohara H, Sohmura T et al (2004) Dentoalveolar growth of patients with complete unilateral cleft lip and palate by early two-stage Furlow and push-back method: preliminary results. Cleft Palate-Craniofacial J. https://doi. org/10.1597/03-020.1

Koehler SA, Brown PA (2009) Forensic epidemiology, 1st edn. CRC Press, Boca Raton

Manigandan T, Sumathy C, Elumalai M et al (2015) Forensic radiology in dentistry. J Pharm Bioallied Sci 7:S260-S264. https://doi.org/10.4103/0975-7406.155944 
Marques J, Musse J, Caetano C et al (2013) Analysis of bite marks in foodstuffs by computer tomography (cone beam (T)--3D reconstruction. J Forensic Odontostomatol 31:1-7

Mian AS, Bennamoun M, Owens R (2006) Three-dimensional model-based object recognition and segmentation in cluttered scenes. IEEE Trans Pattern Anal Mach Intell. https://doi.org/10.1109/TPAMI.2006.213

Pramod JB, Marya A, Sharma V (2012) Role of forensic odontologist in post mortem person identification. Dent Res J (Isfahan) 9:522-530

Pretty IA, Sweet D (2001) A look at forensic dentistry - Part 1: The role of teeth in the determination of human identity. Br Dent J 190:359

Priyanka K, Prasad B (2015) Teeth in fire - morphologic and radiographic alterations: an in vitro study. J Forensic Res 06:1-4. https://doi.org/10.4172/ 2157-7145.1000277

Thali MJ, Braun M, Markwalder TH et al (2003) Bite mark documentation and analysis: the forensic 3D/CAD supported photogrammetry approach Forensic Sci Int 135:115-121

Verma AK, Kumar S, Rathore S, Pandey A (2014) Role of dental expert in forensic odontology. Natl J Maxillofac Surg 5:2-5. https://doi.org/10.4103/0975-5950. 140147

Williams J, Bennamoun M (2001) Simultaneous registration of multiple corresponding point sets. Comput Vis Image Underst. https://doi.org/10. 1006/cviu.2000.0884

Williams JA, Bennamoun M, Latham S (2003) Multiple view 3D registration: a review and a new technique

Xiao G, Ong SH, Foong KWC (2007) 3D registration of partially overlapping surfaces using a volumetric approach. Image Vis Comput. https://doi.org/10 1016/j.imavis.2006.07.006

Zhang Z (2014) Iterative closest point (ICP). In: Ikeuchi K (ed) Computer Vision: A Reference Guide. Springer US, Boston, pp 433-434

\section{Publisher's Note}

Springer Nature remains neutral with regard to jurisdictional claims in published maps and institutional affiliations.

\section{Submit your manuscript to a SpringerOpen ${ }^{\circ}$ journal and benefit from:}

- Convenient online submission

- Rigorous peer review

- Open access: articles freely available online

- High visibility within the field

- Retaining the copyright to your article

Submit your next manuscript at $\boldsymbol{\nabla}$ springeropen.com 